high incidence of delayed development, motor incoordination, behavioral, and speech deficits in children with opsoclonus-myoclonus syndrome (Hammer MS, Larsen MB, Stack CV. Pediatr Neurol 1995;13:21-24; Papero PH et al. Dev Med Child Neurol 1995;37:915932; Ped Neur Briefs March \& Aug 1995, Jan 1996).

\title{
DOPA-RESPONSIVE MOTOR DISORDER WITH SEPIAPTERIN REDUCTASE DEFICIENCY
}

The clinical findings in 7 children from Malta, at first suspected to have cerebral palsy and later diagnosed with sepiapterin reductase deficiency, are reported from Great Ormond Street Hospital, London, UK, and St Luke's Hospital, University of Malta. All had early motor delay with diurnal variation and cognitive impairment. Oculogyric crises occurred from an early age in 6 , hypotonia followed by dystonia in 5 , chorea in 4 , bulbar involvement in 3, and Parkinsonian tremor in 2. Treatment with L-dopa was started at ages 1 to 10 years in doses of 1.5 to $4 \mathrm{mg} / \mathrm{kg} / \mathrm{day}$, and the response was dramatic, except for aggravation of chorea. Improvement was obtained predominantly in motor function and control of oculogyric crises, but cognitive function and learning remained moderately impaired. A worsening of symptoms in hot weather was relieved by an increase in dose of Ldopa. All had a novel mutation in the tetrahydrobiopterin pathway involving sepiapterin reductase, with autosomal recessive inheritance. None had an abnormality in the gene encoding guanosine triphosphate cyclohydrolase 1 (GTPCH1), consistent with the autosomal dominant Segawa disease. (Neville BGR, Parascandalo R, Farrugia R, Felice A. Sepiapterin reductase deficiency: a congenital dopa-responsive motor and cognitive disorder. Brain October 2005;128:2291-2296). (Professor BGR Neville, The Wolfson Centre, Mecklenburgh Square, London WC1N 2AP, UK).

COMMENT. This autosomal recessive, dopa-responsive congenital motor disorder, characterized by dystonia with diurnal variation and dramatic response to L-dopa, resembles in some respects the autosomal dominant GTPCH1, dopa responsive deficiency known as Segawa's disease. Unusual features include a congenital onset, early hypotonia and frequent bulbar involvement, atypical manifestations of Segawa's disease (Ann Neurol 2003;54(Supp 6);S32-45). Both conditions are often incorrectly diagnosed with unexplained cerebral palsy. The authors recommend that infants with unexplained CP should be screened for sepiapterin reductase deficiency, although outside Malta, the disease is probably very rare. The report adds to a range of cases of dopa-responsive motor disorders and the necessity for a trial of Ldopa when the diagnosis is unclear.

\section{INFECTIOUS DISORDERS}

\section{AUTOANTIBODIES IN POST-EPSTEIN-BARR ACUTE CEREBELLAR ATAXIA}

Eight of 23 patients with acute cerebellar ataxia (ACA) following Epstein-Barr virus (EBV) infection (proven serologically) had increased IgM anti-triosephosphate isomerase (TPI) antibody titers, in a study at Kyorin University, Tokyo, and Kinki University, Osaka, 
Japan. Antibody was also positive in 1 of 45 healthy controls, 3 of 4 patients with EBV without ataxia, and 9 of 67 controls with other diseases (meningitis, spinocerebellar degeneration, and systemic lupus erythematosus). The mean antibody titer was significantly higher in the ACA group compared to others, except for those with EBV without ataxia. Chronological studies showed that the antibody titers correlated with the clinical symptom severity and resolution. (Uchibori A, Sakuta M, Kusunoki S, Chiba A. Autoantibodies in postinfectious acute cerebellar ataxia. Neurology October (1 of 2) 2005;65:1114-1116). (Reprints: Dr Atsuro Chiba, Department of Neurology, Schopol of Medicine, Kyorin University, 6-20-2 Shinkawa, Mitaka, Tokyo 181-8611, Japan).

COMMENT. The chronological change in anti-TPI antibody titer suggests that antibody production is stimulated by the preceding EBV infection and is involved in the cause of ataxia. The cerebellar tissue had the strongest antigenicity to the ACA patients' serum. Other infections associated with acute cerebellar ataxia include varicella (Goldston AS, Millichap JG, Miller RH. Am J Dis Child 1963;106:197-200); and Mycopiasma pneumoniae (Ped Neur Briefs 2004;18:62).

\section{$\underline{\text { VASCULAR DISORDERS }}$}

\section{HERBAL SUPPLEMENTS AND CEREBRAL VASCULOPATHY}

A case of stroke attributed to a herbal energy drink containing sympathomimetic phenylpropanoid compounds is reported in a 21-year-old man treated at the University of Virginia, Charlottesville. Within hours after $250 \mathrm{ml}$ of XS Cranberry-Grape Blast while on a hike, the patient, a type 1 diabetic, developed severe headache, photophobia and hemiataxia. Diffusion-weighted MR imaging revealed an acute brainstem infarction and bilateral subarachnoid hemorrhage. Cerebral angiography showed multiple areas of narrowing and dilatation in distal segments of middle and posterior cerebral arteries. A screen for drugs of abuse was negative. The drink contained a mixture of herbal compounds including ginseng and Chinese magnolia vine. In addition, the patient routinely drank the caffeine containing Red Bull Sugar Free. (Worrall BB, Phillips CD, Henderson KK. Herbal energy drinks, phenylpropanoid compounds, and cerebral vasculopathy. Neurology October (1 of 2) 2005;65:1137). (Reprints: Dr BB Worrall, Department of Neurology, University of Virginia Health System, Box 800394, Charlopttesville, VA 22908).

COMMENT. The patient's diabetes may have increased the risk of vasculopathy and stroke with this high energy, ginseng-containing drink. In a report from Taiwan (Ryu S-J, Chien Y-Y. Neurology 1995;45:829-830; Ped Neur Briefs June 1995), ginseng-associated cerebral arteritis and headache were reported in a 28 -year-old woman who had taken an infusion of ginseng root $(25 \mathrm{mg}$ stewed in rice wine) for fatigue. The patient had never experienced headache following smaller quantities of ginseng (Chinese practice usually recommends 0.5 to $2 \mathrm{gm}$ ). Cerebral angiogram revealed multiple areas of alternating focal constriction and beading in anterior and posterior cerebral arteries and superior cerebellar artery, consistent with arteritis. The headache resolved gradually in 10 days. Experimentation with unusually large doses of ginseng to improve stamina or concentration may carry a risk of headache, vasculitis and stroke. 